\title{
Retraction Note: Reduction of carbon dioxide to oxalate by a binuclear copper complex
}

\author{
Uttam R. Pokharel, Frank R. Fronczek \& Andrew W. Maverick
}

Retraction of: Nature Communications https://doi.org/10.1038/ncomms6883, published online 19 December 2014.

During follow-up work to this Article, the authors discovered that its main claim-namely, that $\mathrm{CO}_{2}$ was reduced to form oxalate-is incorrect. Rather, the detected oxalate is formed by the oxidation of ascorbate in the system, as discussed in a subsequent analysis ${ }^{1}$. Although other results reported therein are valid, the authors wish to retract this Article.

Published online: 25 March 2021

\section{Reference}

1. Khamespanah, F. et al. Oxalate production via oxidation of ascorbate rather than reduction of carbon dioxide. Nat. Commun. 12, https://doi.org/10.1038/s41467-02121817-w (2021). 